Int. J. Morphol.,

33(4):1427-1435, 2015.

\title{
La Vía Biliar Intrahepática. Clasificación Anátomo-Quirúrgica con Base Colangiográfica
}

\author{
Intrahepatic Biliary Ducts. Anatomic and Surgical Classification after Cholangiographic Findings
}

\author{
Vicente Mitidieri* \& Nicolás Ernesto Ottone ${ }^{* * * * * *}$
}

\begin{abstract}
MITIDIERI, V. \& OTTONE, N. E. La vía biliar intrahepática. Clasificación anátomo-quirúrgica con base colangiográfica. Int. J. Morphol., 33(4):1427-1435, 2015.

RESUMEN: Las variaciones en la constitución de la vía biliar son muy frecuentes. Su conocimiento adquiere importancia en distintos procedimientos quirúrgicos, como la colocación de un drenaje en la vía biliar o una colecistectomía. Sin embargo, el cirujano a menudo la visualiza por primera vez durante el acto quirúrgico, y debe lidiar con clasificaciones complejas para poder comprenderlas. El objetivo de este trabajo es presentar una clasificación sencilla y de rápida interpretación. Se analizaron 100 estudios colangiográficos y se realizaron 10 disecciones cadavéricas, como apoyo al estudio colangiográfico. A partir de los resultados obtenidos, se propuso la siguiente clasificación: Conducto hepático derecho "típico", cuando éste reúne la bilis de toda la porción hepática derecha, o "dividido", cuando sus ramas desembocan separadamente en la vía biliar principal. Conducto hepático izquierdo "típico" o "dividido", siguiendo el mismo criterio. Conducto hepático "central", cuando las secciones parasagitales de ambas porciones hepáticas, derecha e izquierda, se reúnen en un solo conducto. Respecto a la confluencia de los conductos biliares, puede clasificarse en "típica" precisamente cuando ambos conductos hepáticos también lo son, "triple confluencia" cuando uno o ambos conductos hepáticos se encuentran divididos y todos ellos confluyen en un punto, o "escalonada" cuando uno de ellos se encuentra dividido y la confluencia se realiza a distinta altura, en ocasiones con algún conducto segmentario. Creemos que esta clasificación resulta de fácil aplicación por su sencillez, permitiendo identificar todas las estructuras de la vía biliar rápidamente aún sin contar con estudios previos, colaborando de esta manera en una cirugía más segura.
\end{abstract}

PALABRAS CLAVE: Vía biliar intrahepática; Colangiografía; Clasificación.

\section{INTRODUCCION}

La vía biliar transporta la bilis elaborada por el hígado hacia el duodeno. Presenta una porción extrahepática, que se extiende desde la cara visceral del hígado hasta su desembocadura en la porción descendente del duodeno, y otra intrahepática, ubicada en el espesor de este órgano a partir de los canalículos originados en cada segmento (CS).

Clásicamente, se describen en el lado derecho dos conductos, anterior y posterior, que reciben la bilis de las secciones hepáticas homónimas, y confluyen para formar el conducto hepático derecho (CHD). El conducto hepático izquierdo (CHI) se forma por la confluencia de los canalículos de los segmentos II, III y IV. Ambos conductos se unen en la cara visceral del hígado para formar el conducto hepático.

Las variaciones en la constitución de la vía biliar intrahepática son muy frecuentes, tanto en lo que hace a la distribución segmentaria de los canalículos como a la confluencia a nivel de la placa hiliar. Esto determina que la utilización de drenajes en la vía biliar, o la partición hepática para transplantes, no siempre obtenga el resultado esperado (Choi et al., 1993).

Healey \& Schroy (1953) sentaron las bases para el estudio de la arborización biliar; con el transcurso del tiempo, la aplicación quirúrgica de estos conocimientos dio lugar a múltiples y complejas clasificaciones. El objetivo de esta presentación es aportar la experiencia obtenida en estudios colangiográficos, disecciones cadavéricas y quirúrgicas, en un intento por simplificar la comprensión de estas variantes, particularmente para los cirujanos generales que en el curso de una colecistectomía deben interpretar la anatomía en pocos minutos mediante el estudio colangiográfico, a los efectos de diagnosticar la indemnidad de la totalidad

\footnotetext{
Profesor Adjunto de Anatomía, Médico Cirujano, Miembro de la Asociación Argentina de Cirugía (MAAC). Facultad de Medicina, Universidad de Buenos Aires, Buenos Aires, Argentina.

** CICO Centro de Investigación en Ciencias Odontológicas, Facultad de Odontología, Universidad de La Frontera, Temuco, Chile.

*** Programa de Doctorado en Ciencias Morfológicas, Facultad de Medicina, Universidad de La Frontera, Temuco, Chile.
} 
del árbol biliar, y prevenir lesiones cuyo devenir suele ser gravoso para el enfermo y el cirujano.

Para la denominación de las distintas secciones y segmentos hepáticos utilizaremos la nomenclatura acordada en Brisbane en el año 2000, en una reunión realizada al efecto, con la participación de los cirujanos hepáticos de todo el mundo (Belghiti et al., 2000; Andriani, 2010).

\section{MATERIAL Y MÉTODO}

Se realizaron 10 disecciones en la Facultad de Medicina de la UBA al efecto de estudiar los atributos de las ramas biliares intrahepáticas para correlacionarlas con los hallazgos colangiográficos.

En todos los casos, se observó a la vía biliar envuelta en un engrosamiento fascial dependiente de la cápsula fibrosa perivascular ("cápsula de Glisson"), de las fascias vasculares del pedículo hepático y del omento menor. Es la denominada "Placa Hiliar", que adhiere íntimamente a las estructuras biliares, y de una manera más laxa a la arteria hepática y la vena porta. Su disección es indispensable para poder visualizar la confluencia de los conductos hepáticos (Fig. 1).

Mediante el relleno de la vía biliar se pudo identificar, en dos cadáveres, un canalículo biliar subvesicular, descrito por Healey \& Schroy, ubicado en el fondo de la fosa de la vesícula biliar a todo lo largo de la misma (Fig. 2).

Luego se analizaron 100 colangiografías. Se llamó "segmentarios" a los canalículos dirigidos a cada segmento, y "subsegmentarios" a sus ramas, evitando nombrarlos como de $1^{\text {er }}, 2^{\text {do }} \mathrm{o} 3^{\text {er }}$ orden ya que esta nomenclatura atribuye un mismo nombre a estructuras de diferente jerarquía anatómica.

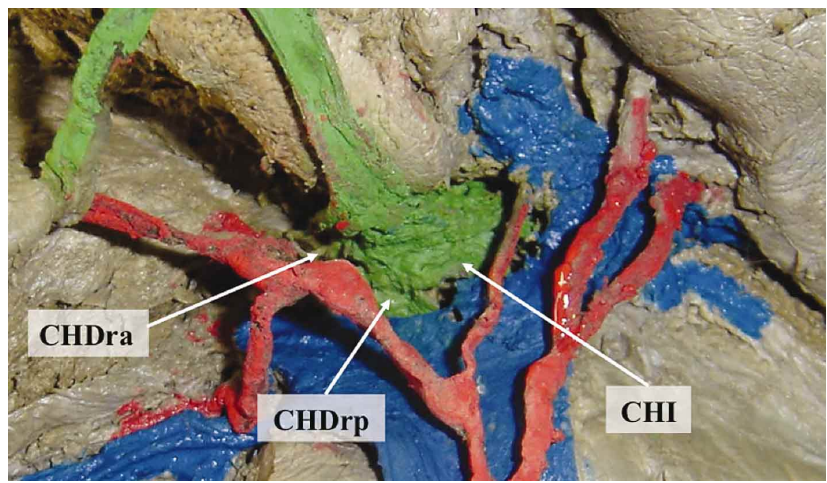

Fig. 1. Visualización de la confluencia de los conductos hepáticos biliares. CHDra conducto hepático derecho, rama anterior; CHDrp, conducto hepático derecho, rama posterior; $\mathrm{CHI}$, conducto hepático izquierdo.

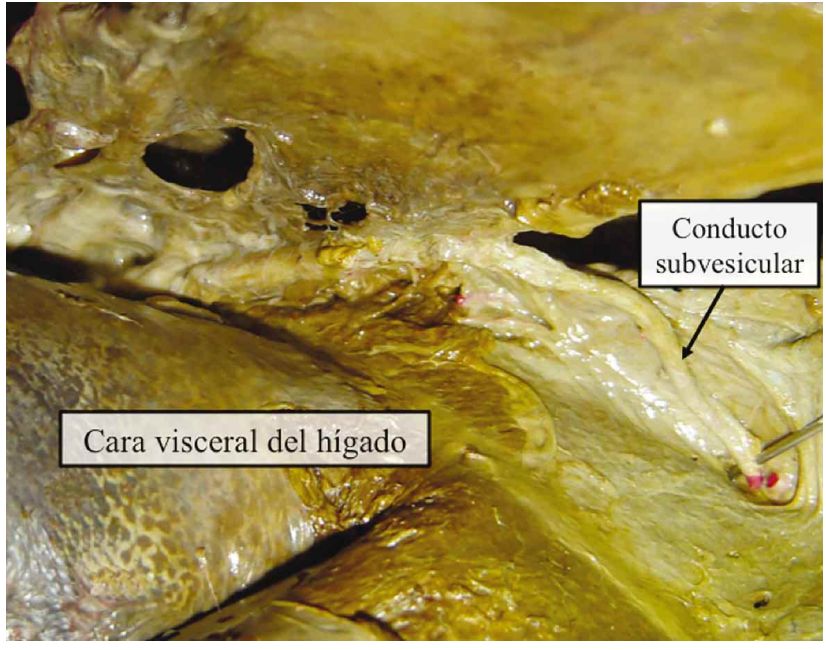

Fig. 2. Identificación de un canalículo biliar subvesicular, ubicado en el fondo de la fosa de la vesícula biliar (descrito por Healey \& Schroi, 1953).

Se utilizó la siguiente metodología (Mitidieri, 2007): Comenzando por el extremo izquierdo del hígado, se visualizan dos canalículos: uno de ellos, superior, corresponde al del segmento II; el inferior, al del segmento III. En el otro extremo, dos canalículos se dirigen caudalmente: el del VI es lateral al del V, que se ubica a su izquierda. Siguiendo a ambos canalículos cranealmente, se reconocen los de los segmentos

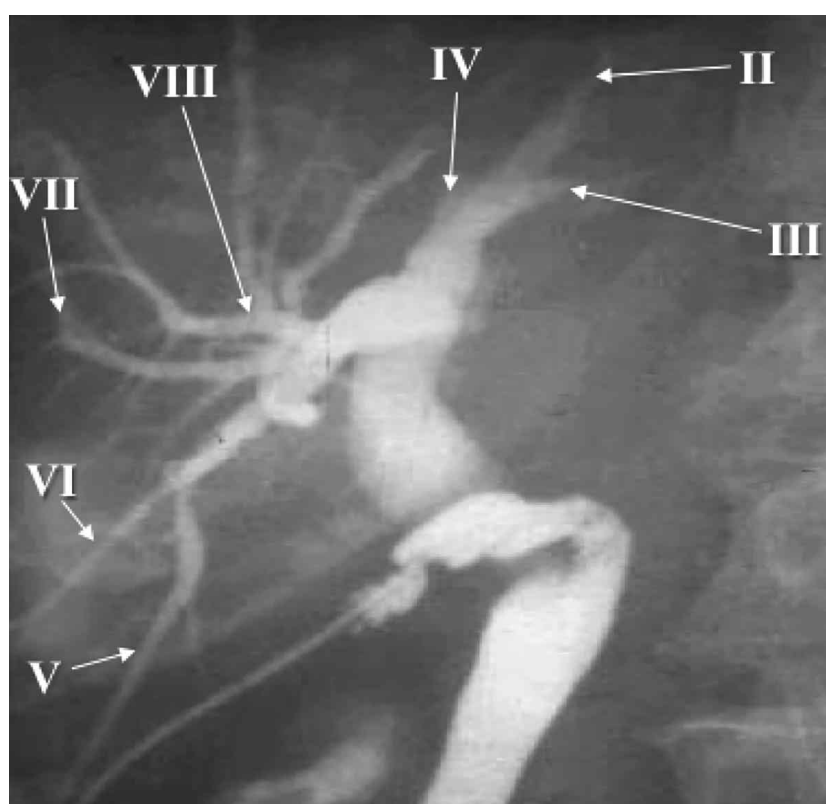

Fig. 3. Estudio colangiográfico en el cual se logran visualizar los canalículos "segmentarios", correspondientes a los segmentos hepáticos II al VIII, los cuales, según la TAI, se denominan: II: Segmento lateral izquierdo; III: Segmento anterior lateral izquierdo; IV: Segmento medial izquierdo; V: Segmento anterior medial derecho; VI: Segmento anterior lateral derecho; VII: Segmento posterior lateral derecho; VIII: Segmento posterior medial derecho. 
VII y VIII. Durante el estudio radioscópico, la rotación del paciente despliega los conductos y se reconocen con mayor certeza. En caso de variaciones en cuanto a la confluencia con los mismos para conformar los ramos posterior y anterior del CHD, debe recordarse que el VIII es el que se ubica más cranealmente sin alcanzar la cara visceral del hígado, siendo su canalículo por lo tanto el de mayor extensión hacia el diafragma. Hacia la izquierda de estos últimos canalículos, se ubica el correspondiente al segmento IV (Fig. 3).

Los canalículos dirigidos al lóbulo caudado presentan una corta longitud, lo que hace difícil identificarlos con certeza, por lo cual fueron excluidos.

\section{RESULTADOS}

Conducto hepático derecho (CHD). El CHD "típico", como se lo concibe clásicamente, fue hallado en un total de 62 casos.

En 20 de ellos hubo variaciones en lo que hace a la formación de los conductos anterior y posterior, que no en todos los casos estaban formados por los canalículos segmentarios (CS) (V+VIII) y (VI+VII) respectivamente: 11 presentaban un CS V desembocando en el conducto posterior; 4 presentaban el canalículo CS VI desembocando en el anterior; en 2 casos, el CS V estaba drenado por 2 conductos, uno de ellos desaguando en el conducto anterior y el restante en el posterior (Fig. 4).

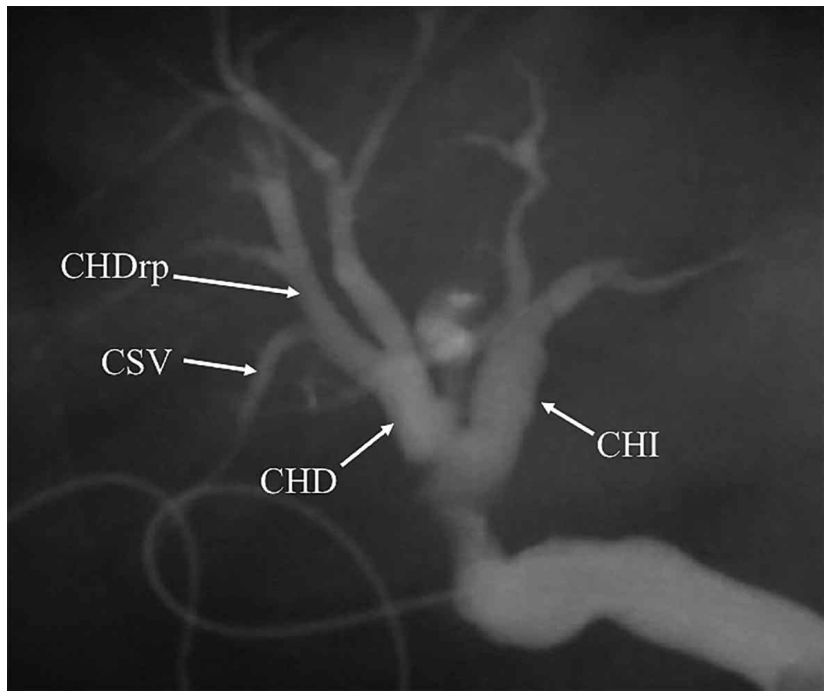

Fig. 4. Estudio colangiográfico donde el canalículo segmentario $\mathrm{V}$ (CS V) es drenado por dos conductos, que drenan en las ramas anterior y posterior del conducto hepático derecho (CHDra y CHDrp, respectivamente). CHI, conducto hepático izquierdo.
En 31 casos, el conducto anterior o el posterior desembocaban separadamente en la vía biliar principal. En estos casos, el CHD se encuentra "dividido" en sus dos componentes, sin llegar a formarse un verdadero CHD que reúna toda la bilis proveniente de la porción hepática derecha (Fig. 5). En 12 de estos 31 casos, la confluencia entre ambos conductos hepáticos derechos y el conducto hepático izquierdo se hacían en un mismo punto, es decir, había una triple confluencia biliar.

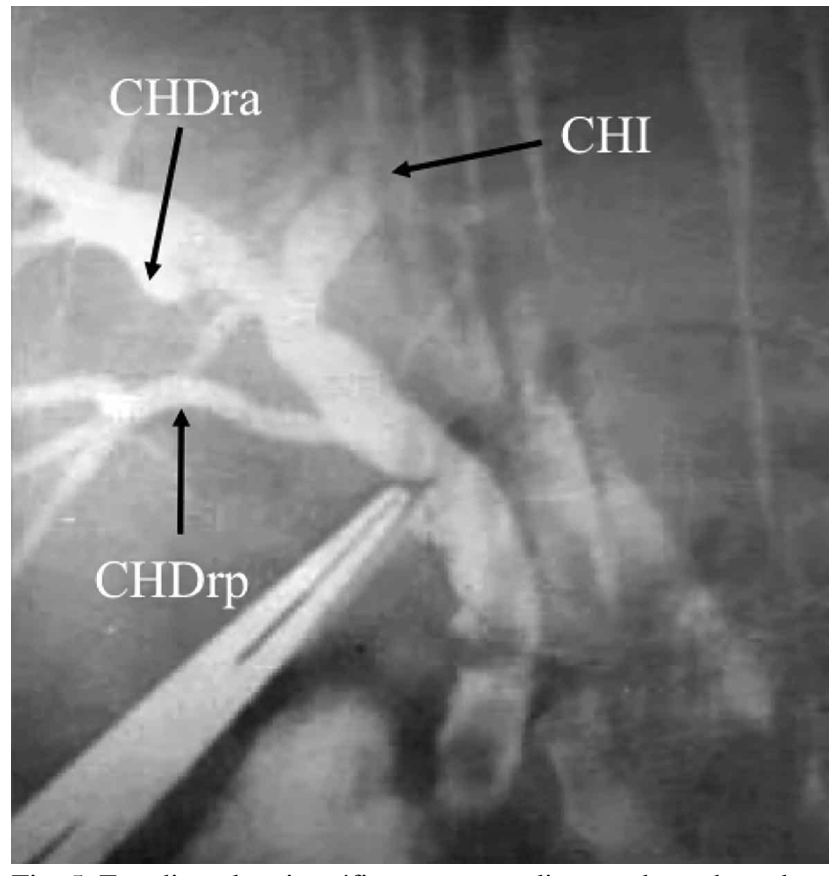

Fig. 5. Estudio colangiográfico correspondiente a desembocadura separada del conducto hepático derecho, rama anterior (CHDra) y el conducto hepático izquierdo, rama posterior (CHDrp). CHI, conducto hepático izquierdo.

Se encontraron 7 casos en los que un canalículo del lóbulo izquierdo del hígado desemboca en un afluente del CHD. Esto permitió clasificar a las variaciones del CHD en 3 variantes (Tabla I).

Respecto a la desembocadura de un canalículo segmentario en la vía biliar principal, se encontraron 3 casos (Fig. 6). No se hallaron conductos hepáticos desembocando directamente en la vesícula biliar.

Conducto hepático izquierdo (CHI) . Un CHI "típico" fue hallado en un total de 83 casos; 44 de ellos presentaban un canalículo que reunía la bilis de los CS II+III, unido luego con el del IV (Fig. 7). En 39 pacientes, el CHI se formaba mediante la unión de los CS III+IV con el del segmento II. Diez de estos casos, cinco en cada grupo, presentaban la desembocadura de uno de estos canalículos muy cercano a la confluencia de ambos conductos hepáticos. 


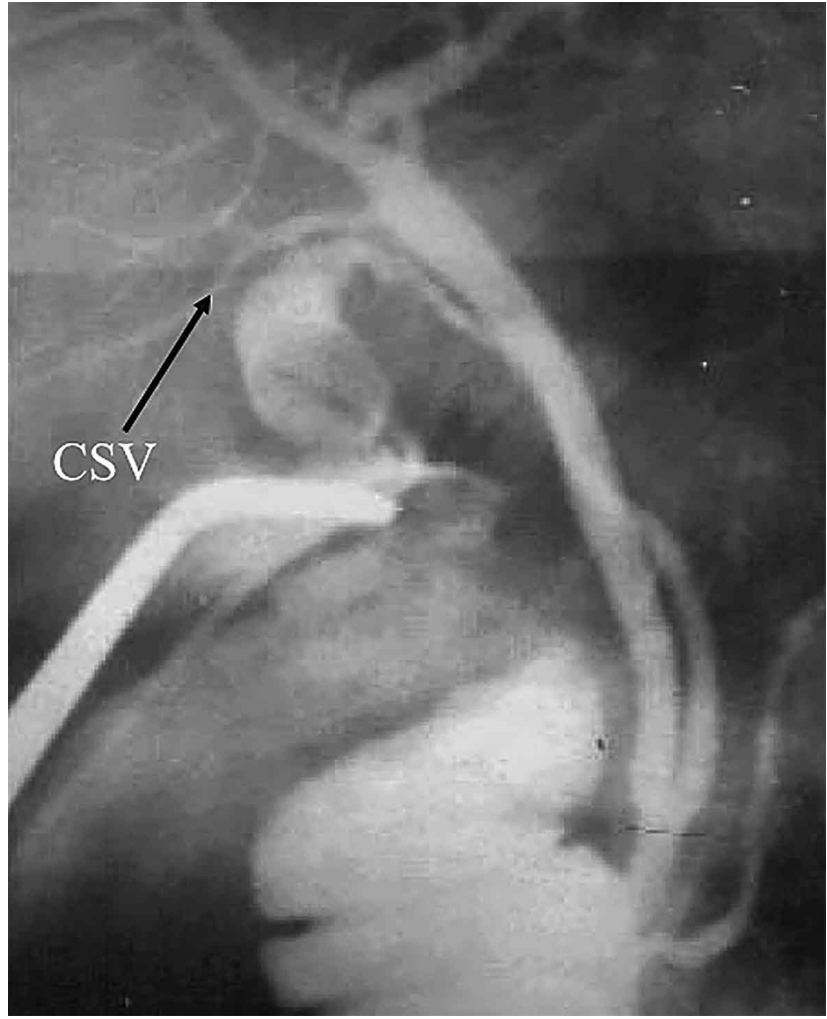

Fig. 6. Estudio colangiográfico que evidencia la presencia de un canalículo subsegmentario (CS V) desembocando en la vía biliar principal

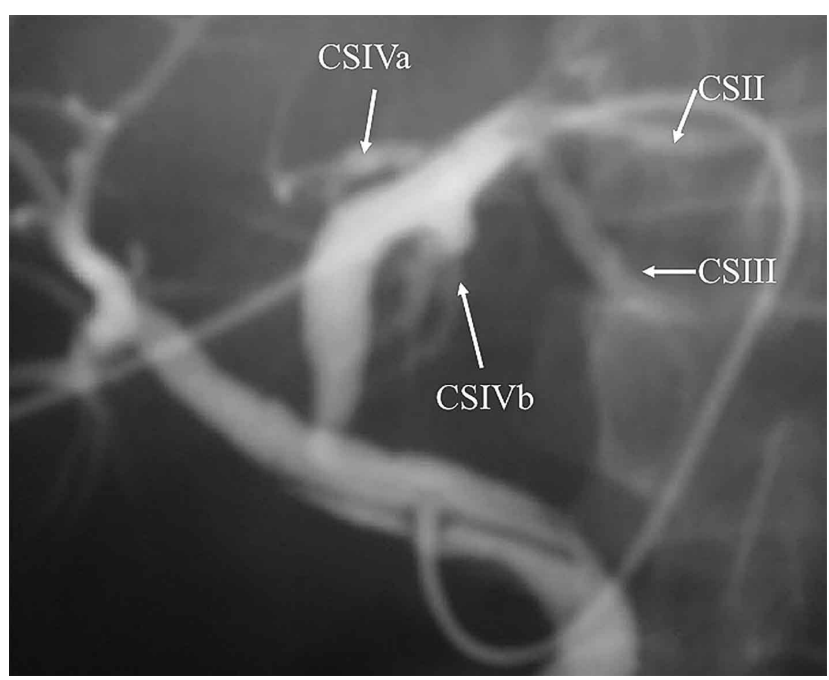

Fig. 7. Estudio colangiográfico orientado al conducto hepático izquierdo ("típico"), donde un canalículo reúne la bilis de los segmentos II y III (CS II y CS III, respectivamente), unidos luego con el canalículo que reúne a los segmentos IVa y IVb (CS IVa y CS $\mathrm{IVb}$, respectivamente).

En 10 casos, dos conductos fueron hallados desaguando directamente en la vía biliar principal (CHI dividido). En
Tabla I. Clasificación de las variaciones el Conducto Hepático Derecho (CHD). CHDra, conducto hepático derecho, rama anterior. CHDrp, conducto hepático derecho, rama posterior.

\begin{tabular}{lc}
\hline Variante 1. Conducto hepático derecho "típico" & $\mathbf{6 2}$ \\
CS V drenando en el CHDrp & 11 \\
CS VI desaguando en el CHDra & 4 \\
CS V dividido, al CHDra y al CHDrp & 2 \\
Variante 2. Conducto hepá tico derecho "dividido & $\mathbf{3 1}$ \\
CHDra y CHDrp drenando en la vía biliar principal & 19 \\
Triple confluencia & 12 \\
Variante 3. Conducto hepático central & 7 \\
\hline
\end{tabular}

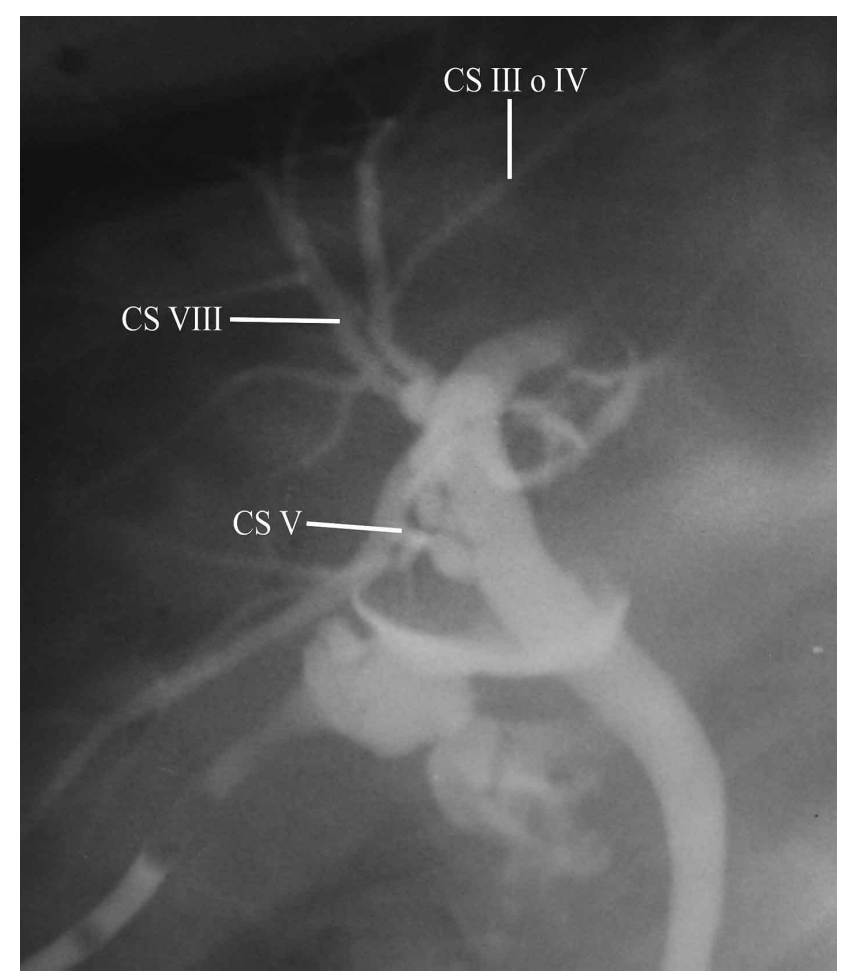

Fig. 8. Estudio colangiográfico orientado al conducto hepático izquierdo dividido, con visualización de los canalículos segmentarios III o IV, V y VIII.

seis, uno estaba conformado por los CS II+III por un lado, y el del segmento IV por el otro; en cuatro casos, se observó un canalículo reuniendo a los CS III+IV, y otro el del CS II. Ambos conductos presentan un trayecto paralelo (Fig 8).

Por último en siete casos hemos hallado que un CS IV desembocaba en el conducto hepático derecho, rama anterior. En estos casos podría decirse que, en rigor, hay ausencia del CHI, ya que ningún conducto reúne toda la bilis de la porción hepática izquierda; hablamos de conducto hepático "central". No hemos hallado canalículos provenientes del lóbulo izquierdo desaguando en el conducto hepático derecho, rama posterior (Fig. 9). 


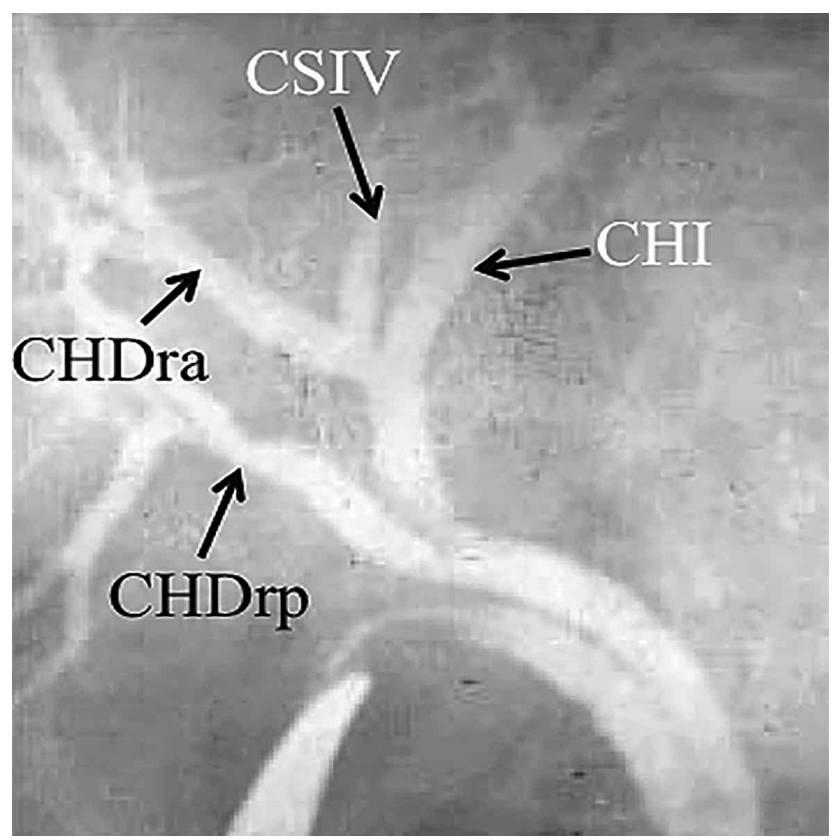

Fig. 9. Estudio colangiográfico donde se visualiza desembocadura del canalículo del segmento IV (CS IV) en el conducto hepático derecho, rama anterior (CHDra). CHI, conducto hepático izquierdo; CHDrp, conducto hepático derecho, rama posterior.

Tabla II. Clasificación de las variaciones del Conducto Hepático Izquierdo (CHI).

\begin{tabular}{lc}
\hline Variante 1. Conducto hepático izquierdo "típico" & $\mathbf{8 3}$ \\
Variante Ia (II+III) + IV & 44 \\
Variante Ib (III+IV) + II & 39 \\
Variante 2. Conducto hepático izquierdo "dividido" & $\mathbf{1 0}$ \\
Variante IIa (II+III) + IV & 6 \\
Variante IIb (III+IV) + II & 4 \\
Variante 3. Conducto hepático central & 7 \\
\hline
\end{tabular}

El CS IV suele ser múltiple. Algunas ramas menores desaguan con mayor frecuencia en el CHD. En estos casos, se tomó como la rama propia de este segmento a la de mayores dimensiones.

Esto nos permitió clasificar a las variaciones del CHI en tres variantes (Tabla II).

\section{DISCUSIÓN}

Conducto hepático derecho y sus afluentes. El CHD se ubica ventralmente a la rama derecha de la vena porta. Se forma por la confluencia de sus dos ramas, anterior y posterior, y luego de un trayecto de aproximadamente un $\mathrm{cm}$, se une con el CHI. Esta descripción se observa entre el 55 y el $72 \%$ de los casos (Healey, 1970; Farina, 1978; Huang et al., 1996; Castaing, 2008; Karakas et al., 2008).

La rama anterior del CHD transcurre a la izquierda y por delante de la rama portal homónima. En el $20 \%$ de los casos puede tener una ubicación retroportal. Recibe un afluente superior, del CS VIII, y otro inferior del CS V. Sin embargo, la rama anterior del CHD aparece como completo únicamente en el 35\% de los casos (Castaing). Hemos hallado al CS V en $11 \%$ de los casos desaguando en la rama posterior del CHD, y en el $2 \%$ mediante dos canalículos separados. En el 3\% de los casos observamos un canalículo segmentario, presumiblemente de este mismo segmento, desaguando en la vía biliar principal.

La rama posterior del CHD es generalmente más larga. Tiene un trayecto horizontal, de dirección anteroposterior, cruzando por detrás de la rama derecha de la vena porta o de su rama anterior. Recibe dos afluentes: uno superior, proveniente del CS VII, y otro inferior, del CS VI. Transcurre por encima de la rama portal en el $80 \%$ de los casos, y por debajo en el resto. Este conducto aparece como completo en el $61 \%$ de los casos. Ocasionalmente, las ramas de los CS VI y VII, pueden desaguar en la rama anterior del CHD (6-10\% de los casos). También puede hacerlo en el CHD (2-4\%) o en el conducto hepático común (2-4\%) (Healey; Farina).

Cabe preguntarse si debería llamarse "hepático común" a la confluencia del CHI y las ramas anterior o posterior del CHD aislados. En rigor, solamente se debería hablar de conducto hepático común una vez que hayan confluido todos los canalículos segmentarios. Preferimos llamar vía biliar principal al eje sobre el cual van desembocando los diferentes conductos una vez producida la confluencia de ramos provenientes de ambas porciones hepáticas, derecha e izquierda. En estos pacientes, no se forma el CHD como la sumatoria de sus ramas anterior y posterior. Podríamos hablar de CHD "dividido". El término "duplicación" no se ajustaría a la realidad, ya que no hay dos conductos hepáticos derechos sino que no se ha conformado siquiera uno.

En aquellos casos en que los canalículos que debieran desembocar en el CHD lo hacen en la vía biliar principal, finalmente no se forma el CHD. Se podría hablar entonces de "CHD ausente" (Castaing). La llegada por separado de conductos segmentarios a la vía biliar principal, configura a su vez una confluencia escalonada ("convergence étagée", "selved confluence"). Choi ha descrito un $19 \%$ de pacientes con drenaje anómalo de un conducto hepático derecho; en un $11 \%$ de ellos, la rama anterior llega al conducto formado por la unión del CHI con la 
rama posterior del CHD, en $6 \%$ de los casos es la rama posterior la que llega a la unión del CHI con la rama anterior del CHD, y en el $2 \%$ el conducto posterior desemboca en el conducto cístico.

En el 10 a $15 \%$ de los casos (Huang et al.; Karakas et al.) la rama anterior del CHD confluye en un mismo punto con la rama posterior y el CHI. Es decir, existe una triple confluencia. Nosotros la hemos hallado en el $12 \%$.

Se ha descripto a la rama posterior del CHD o a sus canalículos segmentarios (VI y VII) desembocando en el conducto cístico. En realidad, debería considerarse que es el conducto cístico el que desagua en un conducto seccional o segmentario, ya que éste tiene una mayor jerarquía anatómica.

También se han descrito pequeños conductos drenando porciones subsegmentarias de parénquima hepático y desembocando en la vesícula biliar. Son los conductos "de Luschka", o "vasa aberrantia", descriptos por Champetier et al. (1982). El término "aberrantia" no parece adecuado, ya que aberrante se refiere a un conducto normal que presenta un trayecto diferente. Deben llamarse "accesorios" ya que drenan una porción no definida de parénquima hepático. La lesión de estos conductos derivaría en pequeñas bilirragias postoperatorias que habitualmente se agotarían espontáneamente. Sin embargo, en las disecciones anatómicas y en más de 1000 colecistectomías consecutivas operadas entre noviembre de 2003 y junio de 2014 utilizando el plano subfascial (Mitidieri et al., 2010) no fue hallado ningún canalículo desembocando en la vesícula biliar. En cambio, se ha descripto con una frecuencia aproximada al $30 \%$ un conducto subvesicular que transcurre entre la cara visceral del hígado y la vesícula biliar (Healey; Farina; Mitidieri; Castaing), denominado "ductus subvesicalis" por Champetier et al. Lo hemos hallado en dos de diez disecciones cadavéricas. Para evitar su lesión, debe recordarse la presencia de una fascia vascular dependiente de la arteria cística que divide en dos al espacio interhepatovesicular: uno de ellos entre la vesícula biliar y la fascia vascular; el otro, entre ésta y la cara visceral del hígado, donde se encuentra el conducto mencionado junto con otras estructuras vasculares y biliares (Mitidieri et al.). Si la disección se realiza entre la superficie de la vesícula y la fascia vascular de la arteria cística, se tiene la certeza de no encontrar este canalículo. La frecuencia de bilirragias en las colecistectomías en que la disección se realiza en íntima relación a la cara visceral del hígado, podría explicarse por la lesión del conducto subvesicular.

Conducto hepático izquierdo y sus afluentes. El drenaje biliar de la porción hepática izquierda también presenta variaciones de importancia. El CS III es generalmente más largo. Se une con el del CS II ya sea a la derecha o a la izquierda de la fisura umbilical. El segmento IV es drenado por un conducto de naturaleza variable. Suele ser múltiple (Couinaud, 1989; Mitidieri \& Lerner, 1993), dado que este sector presenta dos subsegmentos claramente diferenciados, 4a y 4b (Bismuth, 1982). Sin embargo, habitualmente presenta un conducto dominante. Todos estos conductos confluyen para constituir el CHI. En esta investigación se encontró así en el 83\% de los casos.

La unión entre los canalículos segmentarios que drenan la porción hepática izquierda puede no realizarse, y ambos conductos llegar separadamente a la vía biliar principal. Esta variación fue observada entre un $2 \%$ y un $16,8 \%$ de los casos (Couinaud, 1954). Nosotros lo hemos hallado en el 10\%. Farina observó sobre 112 moldes dos conductos hepáticos izquierdos en cinco ocasiones, y tres conductos en dos casos. Resulta razonable decir que, en rigor, no existe $\mathrm{CHI}$, es decir, no existe un conducto único que resuma todo el drenaje biliar de la porción hepática izquierda, sino que se lo encuentra "dividido". En estos casos, el conducto que drena el segmento IV (o IV+III) es anterior respecto al que drena el segmento II (o II+III).

Couinaud en 1989 presentó una completa, pero compleja clasificación, que se puede simplificar con un sentido de aplicación anátomo-quirúrgica en tres categorías (Couinaud, 1954, 1989; Healey; Farina; Bismuth; Champetier et al.; Mitidieri \& Lerner; Huang et al.; Mitidieri; Castaing; Karakas et al.; Mitidieri et al.; Bianchi et al., 2014):

Variante 1: CHI "típico". Se reconocen dos posibilidades: el CS II+III confluye con el CS IV para formar el CHI (variante 1a) o éste se forma por la confluencia de los CS III+IV con el CS II (variante 1b.)

La confluencia puede producirse como ya se ha dicho a la izquierda o a la derecha de la fisura umbilical; en el primer caso, el CHI se presenta como tal en toda la extensión de la placa hiliar; en el segundo, solo habrá un verdadero $\mathrm{CHI}$ en el sector distal a la confluencia, cuya longitud dependerá del nivel en que ésta se produzca. Este dato, de relativa importancia anatómica, adquiere relevancia en el caso de un tumor de la confluencia biliar que, de llegar hasta la unión de estos canalículos, podría dividir el flujo biliar de la porción hepática izquierda. En estos casos, colocar un drenaje en un conducto segmentario no drena la totalidad de la porción hepática izquierda, comportándose como un CHI dividido.

Variante 2: CHI "dividido": uno de los componentes del CHI desemboca en la vía biliar principal, ya sea 
que el CS IV llega en forma separada al CS II + III, (variante 2a) o que el que desemboca directamente en la vía biliar sea el CS II, mientras los CS III y IV forman un conducto común.

En la variante 2, el CHI como tal no existe, ya que no hay conducto alguno que drene la totalidad de la porción hepática izquierda. Se encuentra dividido en sus dos componentes. Es de notar que el CS III drena dos segmentos, mientras que los CS IV o II, pueden eventualmente abarcar a uno solo.

Otro detalle anatómico a tener en cuenta es que el CS IV se ubica en un plano anterior respecto al restante. En estos casos, si se diseca la placa hiliar el conducto ubicado en posición preportal, no será CHI sino al CS IV, produciendo un drenaje biliar insuficiente o, cuanto menos, menor a lo esperado (Couinaud, 1989; Mitidieri \& Lerner; Sugarbaker, 1990).

Variante 3: El CS IV desemboca en una rama proveniente de la porción hepática derecha formando un conducto hepático "central".

En estos casos, un drenaje colocado en el CS IV podría drenar este segmento y parte de la porción hepática izquierda.
Drenaje Biliar del Lóbulo Caudado. Estudiar el drenaje biliar del lóbulo caudado mediante colangiografía es muy difícil, dada la corta longitud de las ramas biliares y la superposición de las mismas con los conductos principales.

Healey \& Schroy afirman que se realiza en el $44 \%$ de los casos mediante un conducto para el proceso caudado, uno para la porción paracava y el restante para el CS I, es decir, un total de tres conductos. En el $26 \%$ de los casos se encuentran dos conductos: uno recolecta el drenaje biliar de la porción paracava y del proceso caudado, mientras que el CS I presenta un conducto aparte. El 30\% restante lo conforman las variaciones.

Los canalículos provenientes del lóbulo caudado y de la porción paracava, llegan a la vía biliar desde su cara posterior, son de poca longitud y calibre, por lo cual son invadidos tempranamente por los tumores de la confluencia biliar.

Cada porción del sector dorsal suele tener un drenaje diferente. Mientras que el proceso caudado en la mayoría de los casos drena hacia el CHD (85\%), el lóbulo caudado lo hace hacia el CHI (93\%). El sector paracava puede drenar prácticamente en iguales proporciones al derecho o al izquierdo.

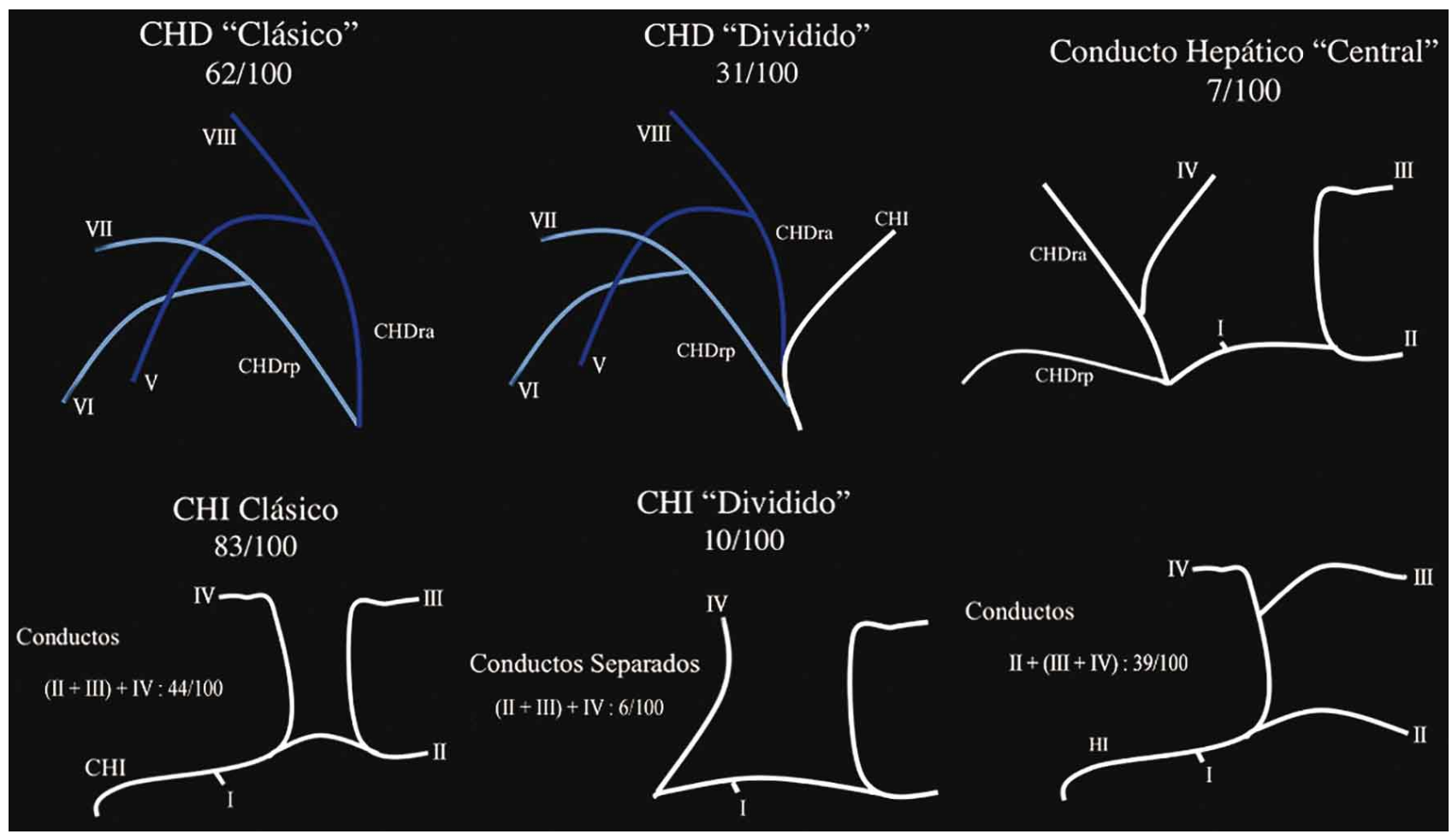

Fig. 10. Clasificación propuesta en esta investigación. 


\section{CONCLUSIONES}

Se han intentado múltiples clasificaciones. Además del trabajo inicial de Healey \& Schroy, se destacan la de Couinaud y Castaign \& Choi. Sin embargo, estas clasificaciones cuando más completas, más complejas.

Tanto el CHD como el CHI pueden conformarse como clásicamente se los concibe (CHD y CHI "típicos"). Sin embargo, sus componentes pueden llegar separadamente a la vía biliar principal, ya sea la rama anterior o la posterior en CHD o las ramas segmentarias en el CHI (conducto "dividido"). Por último, puede haber un conducto "central" que reúna la bilis de ambas secciones paramedianas, mientras que las secciones posterior derecha y lateral izquierda desaguan separadamente.
Respecto a la confluencia, si ambos conductos hepáticos son típicos, la confluencia también será típica.

Si uno de ellos o ambos están "divididos", habrá tres conductos que deben unirse para formar la vía biliar principal. Si lo hacen en un mismo punto, es una "tripe confluencia"; si lo hacen en puntos diferentes, será una "confluencia esclonada", más aún si algún canalículo segmentario desagua directamente a la vía biliar principal.

La clasificación propuesta (Fig. 10), resulta de fácil aplicación por su sencillez. Permite identificar todas las estructuras que conforman el árbol biliar rápidamente en el curso de una colecistectomía aún sin contar con los estudios previos que se utilizan en algunas cirugías de mayor complejidad, y de ese modo reconocer, o mejor aún, evitar su lesión.

MITIDIERI, V. \& OTTONE, N. E. Intrahepatic biliary ducts. Anatomic and surgical classification after cholangiographic findings. Int. J. Morphol., 33(4):1427-1435, 2015.

SUMMARY: Variations in intrahepatic biliary ducts are frequent. Its knowledge is of great importance when facing certain procedures such as drainage or a simple cholecystectomy to avoid iatrogenic lesion or incomplete drainage of the biliary tract. Nevertheless, it is during surgery that the surgeon attempts to see it for the first time, and must deal with complex classifications in order to recognize the ducts. This paper aims to suggest an easy and quick way to interpret and simple classification. 100 cholangiograms were studied and 10 cadaveric specimens were analyzed to support radiologic findings. As a result, we propose the following classification: Right "typical" biliary duct, when all the bile produced in the right hemiliver is drained by a single duct, or "divided" when sectional ducts reach separately the main bile duct. The same applies to the left hepatic ducts, "typical" or "divided". When both paramedian sections are drained by the same duct, there is a "Central" hepatic duct The biliary confluence may be "typical", when both hepatic ducts are also typical, "triple confluence" when one or both hepatic duct are divided and reach the main bile duct in the same place, or "staggered (selved) confluence" (etagée) when one of the ducts is divided and reaches the main bile duct separately from the others. This name is even proper if a segmentary duct reaches the main bile duct. We think this classification is easy to use due to its simplicity, allowing the surgeon to quickly identify each biliary duct and get through the surgery safely.

KEY WORDS: Intrahepatic biliary ducts; Colangiography; Classification.

\section{REFERENCIAS BIBLIOGRÁFICAS}

Andriani, O. C. La torre de Babel, París y Brisbane: Un recorrido sobre sus influencias en la terminología de la segmentación hepática. Rev. Argent. Anat. Online, 1(3):84-9, 2000.

Belghiti, J.; Clavien, P. A.; Gadzijev, E.; Garden, J. O.; Lau, W. Y.; Makuuchi, M. \& Strong, R. W. The Brisbane 2000 Terminology of Liver Anatomy and Resections Terminology Committee of the International Hepato-Pancreato-Biliary Association: Chairman, SM Strasberg (USA). HPB (Oxford), 2:333-9, 2000.

Bianchi, H. F.; Algieri, R. D.; Sanjurjo, D.; Ottone, N. E. \& Fuentes, R. Multiple anatomical variations of the hepatic pedicle: case report and clinical application. Int. J. Morphol., 32(3):782$5,2014$.
Bismuth, H. Surgical anatomy and anatomical surgery of the liver. World J. Surg., 6(1):3-9, 1982.

Castaing, D. Surgical anatomy of the biliary tract. $H P B($ Oxford), 10(2):72-6, 2008.

Champetier, J.; Davin, J. L.; Letoublon, C.; Laborde, Y.; Yver, R. \& Cousset, F. Aberrant biliary ducts (vasa aberrantia): Surgical implications. Anat. Clin., 4(2):137-45, 1982.

Choi, B. I.; Takayasu, K. \& Han, M. C. Small hepatocellular carcinomas and associated nodular lesions of the liver: pathology, pathogenesis, and imaging findings. AJR Am. J. Roentgenol., 160(6):1177-87, 1993. 
Couinaud, C. Lobes et segments hépatiques. Notes sur l'architecture anatomique et chirurgicale du foie. Presse Med., 62(33):709$12,1954$.

Couinaud, C. Exposure of the left hepatic duct through the hilum or in the umbilical of the liver: anatomic limitations. Surgery, 105(1):21-7, 1989.

Farina, C. Estudio de las vías biliares intrahepáticas. Rev. Argent. Cirug., 34:268-71, 1978.

Healey, J. E. \& Schroy, P. C. Anatomy of the biliary ducts within the human liver; analysis of the prevailing pattern of branchings and the major variations of the biliary ducts. AMA Arch. Surg., 66(5):599-616, 1953.

Healey, J. E. Vascular anatomy of the liver. Ann. N. Y. Acad. Sci., 170:8-17, 1970.

Huang, T. L.; Cheng, Y. F.; Chen, C. L.; Chen, T. Y. \& Lee T. Y. Variants of the bile ducts: clinical application in the potential donor of living-related hepatic transplantation. Transplant, Proc., 28(3):1669-70, 1996.

Karakas, H. M.; Celik, T. \& Alicioglu, B. Bile duct anatomy of the Anatolian Caucasian population: Huang classification revisited. Surg. Radiol. Anat., 30(7):539-45, 2008.

Mitidieri, V. \& Lerner, M. Consideraciones acerca de la vía biliar intrahepática y las colangiografías. Publ. Bol. Asoc. Ripl. Anat., 30:81, 1993.

Mitidieri, V. C. Anatomía de la vía biliar. En: Galindo, F. (Ed.). Enciclopedia de Cirugía Digestiva. Tomo IV-437. Buenos Aires, Sociedad Argentina de Cirugía Digestiva, 2007. Disponible en: http://www.sacd.org.ar/ctreintaysiete.pdf

Mitidieri, V.; Mitidieri, A.; Paesano, N. \& Lo Tartaro, M. La lamina vascular de la arteria cística. Aplicación anatomoquirúrgica. Rev. Argent. Anat. Online, 1(3):89-92, 2010.

Sugarbaker, P. H. En bloc resection of hepatic segments 4B, 5 and 6 by transverse hepatectomy. Surg. Gynecol. Obstet., 170(3):250-2, 1990.

\author{
Dirección para Correspondencia: \\ Vicente Mitidieri \\ Médico Cirujano \\ Profesor Adjunto de Anatomía \\ Miembro de la Asociación Argentina de Cirugía (MAAC). \\ Instituto de Morfología J.J. Naón \\ Facultad de Medicina \\ Universidad de Buenos Aires \\ Buenos Aires \\ ARGENTINA
}

Email: vcmitidieri@hotmail.com

Recibido : 15-05-2015

Aceptado: 27-08-2015 